\title{
Introducción
}

\section{La organización social de los cuidados de larga duración en un contexto de austeridad y precariedad}

\author{
Raquel Martínez Buján \\ Universidade da Coruña

\section{Lucía Martínez Virto} \\ Universidad Pública de Navarra \\ <lucia.martinez@unavarra.es>
}

a pertinencia de un monográfico que verse sobre la 'organización social de los cuidados de larga duración’ en España puede explicarse a partir de la confluencia de dos procesos que afectan a la provisión de la asistencia personal y que se han desarrollado en el actual contexto de austeridad política y de recesión económica. En primer lugar, se están produciendo relevantes modificaciones en las bases ideológicas de las directrices de las políticas públicas destinadas a los cuidados de larga duración, que van más allá de la mera reducción de sus presupuestos. En segundo lugar, la precariedad laboral de hombres y mujeres, y la vulnerabilidad social que ésta induce también, advierte de cambios en la forma en la que se distribuyen las tareas de cuidar dentro del grupo familiar. La profundidad con la que se están llevando a cabo estas transformaciones hace pensar que pueden afectar a las formas tradicionales en las que el trabajo de cuidados es distribuido entre el Estado, el mercado, la familia y la comunidad. Por lo tanto, se torna oportuno explorar en estos momentos cuáles son los derroteros de dichas modificaciones y en qué medida están afectando a la configuración de un nuevo modelo de cuidados de larga duración en España.

La llegada de la crisis económica en pleno desarrollo de la Ley de Promoción de Promoción de la Autonomía Personal y Atención a las Personas en Situación de Dependencia (LAPAD) puso sobre la mesa un nuevo orden de prioridades políticas, que cuestionaron su sistema de financiación desde las primeras medidas de austeridad, impulsadas en el Real Decreto Ley 20/2012. Por este motivo, el punto de partida de los artículos de investigación que se incluyen en este número especial es precisamente la situación con la que se encuentra el modelo de cuidados de larga duración desde la aplicación de las medidas de estabilidad presupuestaria. La aminoración en la inversión pública de los cuidados de larga duración que se deriva de dichas directrices ha afectado a las intensidades de algunos de los servicios sociales (como es el caso del servicio de ayuda a domicilio) y ha contribuido a la aparición de nuevas tendencias relacionadas con la promoción de los centros de día y de las prestaciones económicas vinculadas a un servicio (ambos recursos poco desarrollados durante la implantación de la LAPAD). Además, se ha introducido el copago en los servicios ofertados y aparece en el discurso político la necesidad de primar la libertad de elección en la provisión de la asistencia deseada. Todos estos nuevos elementos suscitan varios interrogantes sobre la participación del Estado en el trabajo de cuidados y su capacidad para convertir la asistencia personal en un derecho social. ¿Se está configurando un modelo de cuidados más residual a partir de las restricciones económicas o, por el contrario, se ha conseguido una racionalización del sistema que lo convierte en más profesional? Si éste fuera el caso, ¿se ha producido un debilitamiento en la cobertura de los servicios sociales y se está potenciando la presencia de las empresas privadas que trabajan con la Administración en la provisión de servicios? ¿O más bien se está reforzando un modelo de cuidados de larga duración más familista que en el que en sus orígenes había diseñado la LAPAD? 
En esta línea de análisis, se sitúan los artículos de Julia Montserrat, Rafael Beitia, Fernando Fantova y Manuela Durán. Estos cuatro documentos aportan una amplia y analítica visión sobre cuáles son los cambios que ha experimentado la LAPAD durante la Gran Recesión y en qué medida sus reajustes han modificado tanto el modelo de cuidados que la Ley de Dependencia había implementado en sus inicios como su viabilidad económica. Julia Montserrat nos ofrece un interesante y relevante análisis sobre El nuevo contexto de la protección social de las personas en situación de dependencia, donde explora cómo ha disminuido la protección pública en materia de cuidados de larga duración durante el contexto de crisis económica y evalúa las reformas que ha experimentado la LAPAD en términos de impacto sobre los potenciales demandantes de asistencia personal, y sus consecuencias sobre la creación de un modelo público cada vez más asistencial y con menos intensidad protectora. Este análisis se completa con los datos y reflexiones que aporta Rafael Beitia en La sostenibilidad del sistema para la autonomía y atención a la dependencia durante el trienio 2012-2014: el equilibrio entre costes y financiación, centrados en determinar cuál es la inversión económica que requiere el sistema público de cuidados de larga duración para garantizar su viabilidad, y que introduce el debate sobre las aportaciones de los beneficiarios de dicho modelo de financiación. Por su parte, Manuela Durán, en su artículo Instituciones públicas e instrumentos privados de protección. Una perspectiva de conjunto, detalla cuál era la situación de la red de servicios sociales y de prestaciones económicas antes y después de la entrada en vigor de la LAPAD, para identificar las modificaciones que esta norma ha introducido con respecto al colectivo de personas objeto de protección pública, y la intensidad y cobertura de sus recursos de atención. Fernando Fantova, en Crisis de cuidados y servicios sociales, enuncia algunas propuestas para mejorar la eficacia y eficiencia de las políticas públicas, a partir de una transformación conceptual dirigida a universalizar el concepto de cuidados y superar su tradicional nexo con la atención a las personas en dependencia. Desde ahí, el autor recorre las diferentes formas de cuidado a las personas, arroja luz sobre la potencialidad de la intervención de los sistemas de protección social y se enfrenta al habitual debate sobre las funciones de los servicios sociales en esta materia. Finalmente, a modo de cierre, contrasta su reflexión con el debate sociosanitario en Reino Unido, incorporando matices fundamentales a la organización de los cuidados en España.

De manera paralela a la reformas de la LAPAD, el contexto de crisis económica también ha modificado el sistema español de cuidados con respecto a la implicación de los parientes y a la participación del mercado en la privatización de la asistencia a domicilio. Es decir, las formas en las cuales las familias organizan el trabajo de cuidados, y cómo éste se distribuye entre los parientes y el mercado, parecen adquirir nuevas características desde la destrucción del empleo asalariado y la consiguiente precariedad laboral y social. Por una parte, la interrupción de las trayectorias laborales debido a la destrucción del trabajo remunerado afecta a quienes están disponibles y dispuestos dentro de los hogares a contribuir en las tareas de atención personal, y también repercute en las posibilidades de abonar el copago en caso de acceder a un recurso público. Por otra parte, es probable que el contexto de precariedad laboral y la implementación de medidas de austeridad, como la reducción de la prestación por cuidado familiar (PECEF) o el incremento de tasas o incompatibilidades en algunos servicios de cuidado, también influyan en el proceso de privatización de los cuidados familiares. La literatura académica ha puesto de relieve que, en las últimas dos décadas, se ha experimentado una mercantilización del trabajo familiar de cuidados a través del servicio doméstico, hasta el punto de convertirse en una de las características esenciales del régimen de cuidados español. ¿Supone la destrucción de empleo una distribución más equitativa de las tareas de cuidados entre géneros y generaciones? ¿Se está revertiendo el proceso de privatización a través del servicio doméstico debido a la reducción de los presupuestos familiares? ¿Están regresando a su hogar personas dependientes que estaban recibiendo cuidados institucionales? ¿Existen nuevas propuestas de profesionalización del trabajo de cuidados?

Las contribuciones de este número que indagan sobre la distribución de las tareas familiares de cuidados y su mercantilización mediante la contratación por empleadas de hogar son las de Samara de las Heras, Julia Nogueira y Joseba Zalakain. Samara de las Heras, desde la perspectiva jurídica, presenta un texto titulado Regulación de la responsabilidad de cuidar y su impacto de género, en el que cuestiona cómo las normativas legales que definen los cuidados de larga duración determinan una ideología de cuidados en la que subyace implícitamente quiénes tienen la principal responsabilidad de cuidar y quiénes son los que precisan sus cuidados. Julia Nogueira y Joseba Zalakain, en Discriminación múltiple de las mujeres extranjeras que trabajan en servicios domésticos y de cuidado a dependientes en Euskadi, estudian cómo se ha ido consolidando en los últimos años la actividad del cuidado de personas mayores a domicilio mediante la contratación de servicio doméstico en el que habitualmente trabajan mujeres extranjeras. Asimismo, incorporan un análisis de los cambios que dicha ocupación ha introducido en la organización familiar del cuidado y las condiciones laborales de estas trabajadoras.

Todos estos procesos representan tendencias generales a escala nacional en España, pero es preciso mencionar que su aplicación práctica no ha sido uniforme, sino que ha variado su implementación según las prioridades políticas de cada comunidad autónoma, su realidad sociodemográfica y la desigual incorporación de las mujeres al mercado laboral. Para dar cabida a la 
pluralidad de fórmulas que experimenta la gestión de cuidados a escala regional, este número incluye análisis de caso que minan el concepto ampliamente compartido por la literatura académica de regímenes de cuidados nacionales. De esta manera, se recogen exploraciones sobre los cambios en la gestión de las políticas públicas durante la etapa de recesión económica y su implementación a escala autonómica. Aquí cabría mencionar las aportaciones de Magdalena Díaz, Begoña Elizalde, Nicolás Barbieri, Raquel Gallego, María Teresa Martín, Inmaculada Zambrano, Evangelina Olid y José María Muñoz. Nicolás Barbieri y Raquel Gallego comparan el despliegue e implementación de la Ley de Dependencia en el País Vasco y Madrid en un artículo titulado El despliegue de la Ley de Dependencia en el País Vasco y la Comunidad de Madrid (2007-2012): el margen de la autonomía política, y explican con gran detalle cómo las directrices de la política social que se definen a escala estatal pueden adquirir formas divergentes en su puesta en marcha a escala regional. María Teresa Martín, Inmaculada Zambrano, Evangelina Olid y José María Muñoz complementan este análisis con un artículo titulado Solo lo más urgente: de lo universal a lo asistencial en el modelo de atención pública a la dependencia en Andalucía, que aporta resultados de un sustancioso trabajo de campo que evalúa los cambios que ha experimentado la LAPAD en dicha comunidad desde su implementación, ofreciendo también muy interesantes reflexiones realizadas por los profesionales y técnicos que se ocupan de su gestión y puesta en práctica. Magdalena Díaz y Begoña Elizalde, en Desprofesionalizando la asistencia a domicilio en los cuidados de larga duración: análisis de la reconfiguración del sector en la región de Madrid, analizan los cambios que ha experimentado el servicio público de ayuda a domicilio en esta región, incorporando las modificaciones que ha sufrido a partir de las restricciones económicas y en qué medida han afectado a las condiciones de trabajo de las auxiliares a domicilio que dispensan los cuidados. En este sentido, se introduce el debate del impacto de estos cambios en la profesionalización del sector.

Precisamente, los dos últimos artículos del monográfico valoran la configuración de la profesionalización de los cuidados de larga duración en España. Paloma Moré, con su texto Configuraciones del cuidado de larga duración en España y en Francia: efectos sobre las situaciones concretas de trabajo, expone, de manera comparada entre los dos países, las fórmulas que han seguido en la organización social de los cuidos y cuál ha sido la opción española y sus efectos en términos de empleo. En este sentido, Sara Moreno, Carolina Recio, Vicent Borrás y Teresa Torns, aportan reflexiones muy relevantes en La profesionalización del sector de cuidados. Su artículo está basado en un estudio cualitativo del imaginario social vinculado a las actividades desarrolladas en el sector doméstico y de cuidados, de tal forma que su análisis permite conocer las implicaciones de conceptos abordados en anteriores contribuciones, como la feminización del cuidado, la responsabilidad de cuidar, el desarrollo de normativa reguladora o las respuestas ante la crisis. Con todo ello, sus autores nos dejan ante uno de los mayores retos del sector: su profesionalización en un Estado donde los valores culturales continúan definiendo la práctica de la asistencia personal.

Esperamos que cada una de estas exploraciones nos acerquen a comprender cuáles son las características del modelo de cuidados de larga duración en España y en qué medida se están produciendo cambios en su configuración pública, familiar y mercantil. Sólo un análisis profundo de este sistema podrá inspirar una gestión pública eficiente, una provisión mercantil acorde a las necesidades de las personas cuidadas y un mejor reparto de las tareas de asistencia entre géneros y generaciones. 